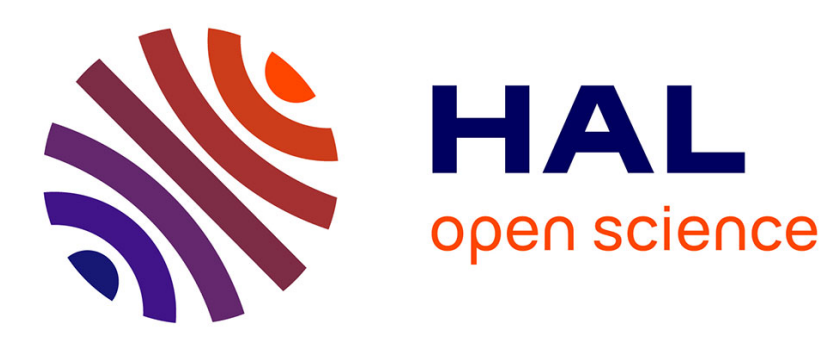

\title{
Micromechanics of Transformation-Induced Plasticity and Variant Coalescence
}

\author{
F. Marketz, F. Fischer, K. Tanaka
}

\section{To cite this version:}

F. Marketz, F. Fischer, K. Tanaka. Micromechanics of Transformation-Induced Plasticity and Variant Coalescence. Journal de Physique IV Proceedings, 1996, 06 (C1), pp.C1-445-C1-454. 10.1051/jp4:1996143 . jpa-00254175

\section{HAL Id: jpa-00254175 https://hal.science/jpa-00254175}

Submitted on 1 Jan 1996

HAL is a multi-disciplinary open access archive for the deposit and dissemination of scientific research documents, whether they are published or not. The documents may come from teaching and research institutions in France or abroad, or from public or private research centers.
L'archive ouverte pluridisciplinaire HAL, est destinée au dépôt et à la diffusion de documents scientifiques de niveau recherche, publiés ou non, émanant des établissements d'enseignement et de recherche français ou étrangers, des laboratoires publics ou privés. 


\title{
Micromechanics of Transformation-Induced Plasticity and Variant Coalescence
}

\author{
F. Marketz*, F.D. Fischer*,** and K. Tanaka*** \\ * Christian Doppler Laboratory for Micromechanics of Materials, University for Mining and Metallurgy, \\ 8700 Leoben, Austria \\ ** Institute of Mechanics, University for Mining and Metallurgy, 8700 Leoben, Austria \\ *** Tokyo Metropolitan Institute of Technology, Department of Aerospace Engineering, Asahigaoka 6-6, \\ Hino-Tokyo, Japan
}

\begin{abstract}
Quantitative micromechanics descriptions of both transformation-induced plasticity (TRIP) associated with the martensitic transformation in an Fe- $\mathrm{Ni}$ alloy and of variant coalescence in a $\mathrm{Cu}$ - $\mathrm{Al}-\mathrm{Ni}$ shape memory alloy are presented. The macroscopic deformation behavior of a polycrystalline aggregate as a result of the rearrangements within the crystallites is modelled with the help of a finite element based periodic microfield approach. In the case of TRIP the parent $\rightarrow$ martensite transformation is described by microscale thermodynamic and kinetic equations taking into account internal stress states. The simulation of a classical experiment on TRIP allows to quantify the Magee-effect and the Greenwood-Johnson effect. Furthermore, the development of the martensitic microstructure is studied with respect to the stress-assisted transformation of preferred variants. In the case of variant coalescence the strain energy due to internal stress states has an important influence on the mechanical behavior. Formulating the reorientation process on the size scale of self-accommodating plate groups in terms of the mobility of the boundaries between martensitic variants the macroscopic behavior in uniaxial tension is predicted by an incremental modelling procedure. Furthermore, influence of energy dissipation on the overall behavior is quantified.
\end{abstract}

\section{INTRODUCTION}

The improvement and development of low-alloy TRIP-steels and shape memory alloys in the viewpoint of desired properties for special applications are based on ideas to judiciously utilize the mechanical properties of martensitic transformations (MT). Subtle aspects of microstructural changes such as the formation of the martensitic phase from the parent phase material or the reorientation of the martensitic phase upon stressing reveal themselves directly in important features of the associated thermomechanical behavior. Catering to a combined viewpoint of material design and MT it is obviously required to achieve an understanding of MT mechanisms and their consequences on the macroscopic mechanical behavior. If it were possible to achieve a controlled microstructural rearrangement under defined thermomechanical loading conditions one would be able to predict a microstructure with the desired macroscopic response under service conditions. Therefore, the development of numerical tools capable to place the microstructure-mechanics connection in the case of MT on a quantitative basis is a growing research thrust. Modelling the mechanical behavior of polycrystalline alloys under martensitic transformation or reorientation processes demands a framework encompassing the interdisciplinary character of the subject with respect to the thermodynamics, crystallography, kinetics and mechanics of phase transformations.

Continuum micromechanics is a helpful tool to treat material problems if the consideration of microstructure is involved [1]. Therefore, our computational micromechanics concept gives much support to the idea of addressing the role of internal stresses on the microstructural changes, the spatial variations of stress and strain fields and couplings related to size scales. Within this article we present a quantitative micromechanics study on TRIP in the case of an Fe-Ni alloy to understand MT as a microscale deformation mechanism causing plastic deformations due to accommodation of the shape changes associated with MT. The non-isothermal creep test [2] is the subject of a micromechanical simulation. Secondly, the reorientation process of the martensitic phase in a $\mathrm{Cu}-\mathrm{Al}-\mathrm{Ni}$ shape memory alloy upon stressing as a step of the one-way shape memory effect will be subject of a micromechanics treatment. 


\section{MICROMECHANICS Of TRANSFORMATION-INDUCED PLASTICITY}

\subsection{Experimental Evidence}

TRIP in the case of MT is attributed at one hand to the orientation of the martensitic phase and, on the other hand, to microscale plastic deformations as a result of the accommodation of the transformation shape change which is the source of transformation-induced internal stress states. If the material under consideration, an Fe-Ni alloy, undergoes MT in an externally-applied stress field $\Sigma$ with a stress level even lower than the yield stress of the parent phase, the effect of $\Sigma$ leads to non-elastic macroscopic strains. In the case of $\mathrm{Fe}-\mathrm{Ni}$ alloys the experimental evidence for TRIP has been given recently by Gautier et al. [2] performing a non-isothermal creep test. The MT occurs by undercooling under the martensite start temperature under constant $\Sigma$. The interaction of stress with the MT mechanism causes the stress-assisted transformation of martensitic variants with preferred orientation. The corresponding macroscopic deformations due to the MT acting as a deformation mechanism is known as the orientation effect (OE) or Magee-effect [3], [4]. The contribution of the plastic strains resulting from the internal stress states to the macroscopic deformation is denoted as the accommodation effect (AE) or Greenwood-Johnson effect. The micromechanical simulation of the non-isothermal creep test is carried out to compute the macroscopic deformation of a specimen in dependence of the level of $\Sigma$ and the martensitic volume fraction $\xi_{M}$ transformed. In the experiment the $\mathrm{OE}$ and the $\mathrm{AE}$ appear coupled whereas the numerial results permit to quantify the respective contributions to the macroscopic deformation. Furthermore, the development of martensitic microstructure in individual grains $k$ of a polycrystalline aggregate can be studied by computing the values for the volume fractions $\xi_{M, j}^{k}$ of variants of martensite forming along definite crystallographic planes.

\subsection{Description of Martensitic Microstructure}

In the case of an Fe-31Ni alloy the martensitic phase appears with a plate-type morphology emerging along habit planes which are crystallographic planes defined with respect to the parent phase $f c c$-lattice. Let us define the mesodomain with volume $V$ as a polycrystalline aggregate consisting of a finite number of $\mathrm{N}$ crystallites. The phenomenological theory of crystallography of MT [5] (WLR-theory) predicts 24 variants in a crystallite with a $\left\{\begin{array}{llll}3 & 14 & 10\end{array}\right\}_{f c c}$ habit plane type in Fe-Ni alloys with high nickel contents. The MT is accompanied by a change in the shape of a variant transformed. This deformation mode is an invariant plane strain. The deformation gradient $\mathbf{F}_{T, j}^{k}$ and the corresponding transformation strain tensor $\epsilon_{T, j}^{k}$ due to transformation of one variant $j$. of 24 possible within a crystallite $k$ with the orientation described by a set of Euler angles $g_{k}=\left\{\varphi_{1}^{k}, \Phi^{k}, \varphi_{2}^{k}\right\}$ within a polycrystalline aggregate are written as

$$
\mathbf{F}_{T, j}^{k}=\mathbf{I}+m\left(\underline{d}_{j}^{k} \otimes \underline{n}_{j}^{k}\right) \quad \text { and } \quad \epsilon_{T, j}^{k}=\frac{1}{2}\left(\mathbf{F}_{T, j}^{k} \mathbf{F}_{T, j}^{k}-\mathbf{I}\right) .
$$

$\underline{n}_{j}^{k}$ is the habit plane unit normal vector, $\underline{d}_{j}^{k}$ the direction and $m$ the magnitude of the transformation shape change. The mechanism for the formation of martensite is taken to be described by the WLR-theory. A "frozen" martensitic domain structure is assumed since in the experiment simulated $\Sigma$ is kept constant and the domain structure formation is mainly affected by the transformation mechanism itself. In a partially transformed state at a temperature $T$ between the martensite start temperature $M_{s}(\Sigma)$ and martensite finish temperature $M_{f}(\Sigma)$ certain variants have transformed with volume fractions $\xi_{M, j}^{k}$. The martensitic microstructure within this framework is described by transformation strain tensors and the volume fractions of variants. The macroscopic transformation strain tensor $\mathbf{E}_{T}$ as an average over the mesodomain resulting from the MT as a deformation mechanism and the macroscopic volume fraction of martensite $\xi_{M}$ are calculated as

$$
\mathbf{E}_{T}=\sum_{k=1}^{N} \sum_{j=1}^{24} \xi_{M, j}^{k} \epsilon_{T, j}^{k} \quad \text { and } \quad \xi_{M}=\sum_{k=1}^{N} \sum_{j=1}^{24} \xi_{M, j}^{k}
$$

$\mathbf{E}_{T}$ kinematically describes the $\mathrm{OE}$. It depends on the martensitic microstructure, that means, on the sequence of transforming variants and their volume fractions. 


\subsection{Description of Parent $\rightarrow$ Martensite Transformation}

The macroscopic deformation behavior is a result of the sequence of transformation variants within the temperature range $M_{s}(\Sigma) \geq T \geq M_{f}(\Sigma)$. A condition for the formation of variants at a certain temperature taking into account the effect of both $\Sigma$ and the internal stress state $\sigma_{\text {int }}$ can be derived within a continuumthermodynamics framework for MT [6]. For the polycrystalline mesodomain the state of the material can be described by an explicit formulation of a thermomechanical potential suggesting the form of Gibbs free energy of thermodynamics. For a detailed formulation in the case of elastic-plastic material behavior we refer to Refs. [6] and [7]. A condition for the formation of a variant $j$ within a crystallite $k$ can be derived from Gibbs free energy resulting as a condition for stress-assisted transformation in terms of thermodynamic forces. The sum of the temperature-dependent chemical driving force $\Delta \phi_{C}^{V}(T)$ defined per unit volume and the mechanical driving force for MT [3], [9], depending both on $\Sigma$ and $\sigma_{\text {int }}$, has to exceed an energy barrier $\Delta \phi_{\text {crit }}^{V}$

$$
\Delta G_{j}^{V k}=\Delta \phi_{C}^{V}+\Delta G_{\sigma, j}^{V k}=\Delta \phi_{C}^{V}+\sigma^{k}: \epsilon_{T, j}^{k} \geq \Delta \phi_{c r i t}^{V} .
$$

$\sigma^{k}$ is the average stress state within a crystallite $k$ which depends on $\Sigma, T$ and the so called pattern of internal rearrangement [10]. It is calculated by application of the finite element method. The formation of martensitic variants occurs by stress-assisted transformation. If the potency of nucleation sites can be equivalently expressed in terms of a threshold thermodynamic force for their operation [12], $\Delta \phi_{\text {crit }}^{V}=\Delta \phi_{C}^{V}\left(M_{s}\right)$ can be regarded as a type of nucleation barrier. $\Delta \phi_{C}^{V}\left(M_{s}\right)$ is the change in the free energy of the stress-free phases at the martensite start temperature $M_{s}$ for $\Sigma=0$. In the proposed continuum formulation presented above the crystallite is treated as a continuum with a transformation behavior taken to be governed by heterogeneous, classical nucleation with a semicoherent state of strain relaxation. The transformation condition implies an invariant plane strain assumption for a critical martensitic nucleus [11]. In the continuum-thermodynamics framework presented here it is difficult to implement the growth behavior of martensitic plates via simple microscale constititive formulations. So, we introduce a kinetic relation in incremental form for the volume fractions $\xi_{M, j}^{V}$. $f_{k}$ is the volume fraction of a crystallite so that $\sum_{k=1}^{N} f_{k}=1$. For a given increment of martensitic fraction within a crystallite $\dot{\xi}_{M}^{k}=f_{k} \dot{\xi}_{M}$ the increment for a variant $\dot{\xi}_{M, j}^{k}$ is taken to be proportional to the thermodynamic force $\Delta G_{j}^{V k}$ for its formation,

$$
\dot{\xi}_{M, j}^{k}=\frac{\Delta G_{j}^{V k}}{\sum_{j=1}^{24} \Delta G_{j}^{V k}} \dot{\xi}_{M}^{k} \quad \text { if } \quad \Delta G_{j}^{V k}>\Delta \phi_{c r i t}^{V}
$$

The sum is taken only for thermodynamic forces corresponding to activated variants so that

$$
\dot{\xi}_{M, j}^{k}=0 \quad \text { if } \quad \Delta G_{j}^{V k} \leq \Delta \phi_{c r i t}^{V} .
$$

The transformation condition and the microscale kinetic equation describe the transformation progress on the size scale of a crystallite.

\subsection{Micromechanical Modelling Framework}

The mesodomain under consideration is modelled as a polycrystalline aggregate consisting of $\mathrm{N}=192$ crystallites each of which having the shape of a regular hexagon. The finite element discretisation is carried out the way that each crystallite is discretized into six triangular finite elements with quadratic shape functions. A two-dimensional general-plane-strain idealization is assumed. The mesodomain is considered as a representative volume element. The polycrystalline microstructure is repeated periodically due to application of antisymmetry boundary conditions [7]. The orientations $g_{k}$ of the $\mathrm{N}$ crystallites are randomly chosen to avoid texture effects. The elastic properties of the crystallites are taken dependent on the orientation of the crystallites.

The micromechanical simulation of the non-isothermal creep test is carried out as follows: In the fully parent phase state, $\xi_{M}=0, T>M_{s}(\boldsymbol{\Sigma})$, the mesodomain is loaded in uniaxial tension in the elastic range. The externally-applied stress $\Sigma$ is kept constant during the transformation progress in the range 
$M_{s}(\Sigma) \geq T \geq M_{f}(\Sigma)$. For the MT driven by a variation of temperature the macroscopic volume fraction of martensite $\xi_{M}$ can be related to temperature by a macrokinetics relation derived with the help of the Magee-assumption [4],

$$
\xi_{M}(T, \Sigma)=1-\exp \left[-b \int_{M_{s}(\Sigma)}^{T} \frac{\partial \Delta \phi_{C}^{V}(T)}{\partial T} d T\right],
$$

b is a material constant. $M_{s}(\Sigma)$ is calculated following the concept of Patel and Cohen [3] which has been extended to tridimensional stress states in Ref. [9]. The elastic anisotropy of the crystallites causes a non-homogeneous stress state which develops due to the compensation of the deformation incompatibilities between the crystallites. Therefore, the martensite start temperature varies from crystallite to crystallite as analysed in Ref. [9]. For the simulation here we assume that the MT starts at $M_{s}(\Sigma)$ within each crystallite but for the formation of variants the effect of internal stress states is considered. The incremental modelling procedure for transformation increments $\Delta T \ll M_{f}(\Sigma)-M_{s}(\Sigma)$ works as follows. Starting at $M_{s}(\Sigma)$ the mesodomain is cooled by a small amount $\Delta T$ resulting in an increment $\Delta \xi_{M}$. A homogeneous temperature distribution within the mesodomain is assumed, that means, the transfer of the latent heat associated with MT occurs fast enough to neglect its effect on the transformation progress. Taking the transformation condition eq.(3) and the microkinetics relation eq.(4) the average transformation strain tensor for a crystallite $k$ is calculated by

$$
\dot{\mathbf{E}}_{T}=\sum_{k=1}^{N} \sum_{j=1}^{24} \dot{\xi}_{M, j}^{k} \epsilon_{T, j}^{k}
$$

For each crystallite the transformation process is kinematically described for an increment $\Delta T$. In the micromechanical model the transformation strains are imposed incrementally. The local stress state $\sigma$ results from $\Sigma$, the elastic anisotropy of the crystallites and transformation-induced internal stresses. The local strain tensor $\epsilon$ is composed of elastic, plastic and transformation strains. For the constitutive behavior in the non-elastic range the $J_{2}$-flow theory with linear isotropic hardening is applied. The yield stress $\sigma_{Y}\left(\xi_{M}\right)$ and the plastic tangent modulus $E_{p}\left(\xi_{M}\right)$ are assumed to obey a linear rule of mixture with respect to the volume fraction transformed. For material data chosen here we refer to Ref. [8]. For each transformation increment $\Delta \xi_{M}$ the periodic microfield approach allows the computation of the corresponding increment of macroscopic plastic strain $\dot{\mathbf{E}}_{P}$ quantifying the accommodation effect. At the end of each increment the average stress tensor $\boldsymbol{\sigma}^{k}$ for each crystallite is calculated and then the next transformation increment is defined by applying eq.(3), eq.(4) and eq.(7). Such a modelling framework allows to consider the interaction of stress, strain and the transformation mechanism on the size scale of the crystallites and to quantify the effect on the macroscopic behavior with the micro-macro transition.

\subsection{Results and Discussion}

In Figure la the TRIP-strain $\epsilon_{T P}$ is depicted for various levels of applied tensile stress. The results agree not only qualitatively but also quantitatively with the experiments [2]. The TRIP-strain $\epsilon_{T P}$ is composed of $\epsilon_{T}$ and $\epsilon_{P}$ related to the $\mathrm{OE}$ and the AE. The contribution of $\epsilon_{T}$ to $\epsilon_{T P}$ in percent $t=\left(\epsilon_{T} / \epsilon_{T P}\right) \times 100$ is depicted in Figure 1b. As reported in [2] the $\mathrm{OE}$ is the dominating mechanism for small fractions $\xi_{M}<0.2$. For higher fractions the $\mathrm{OE}$ is nearly constant. The AE increases with increasing level of applied stress but remains smaller than the $\mathrm{OE}$. The $\mathrm{OE}$ does not vanish even if transformation-induced internal stress states develop. If the applied stress level is near the yield stress $\sigma_{Y, A}$ of the parent phase, plastic deformations can occur at small volume fractions $\xi_{M}$. The AE decreases with increasing $\xi_{M}$ since the yield stress of the two-phase system increases with increasing $\xi_{M}$. 

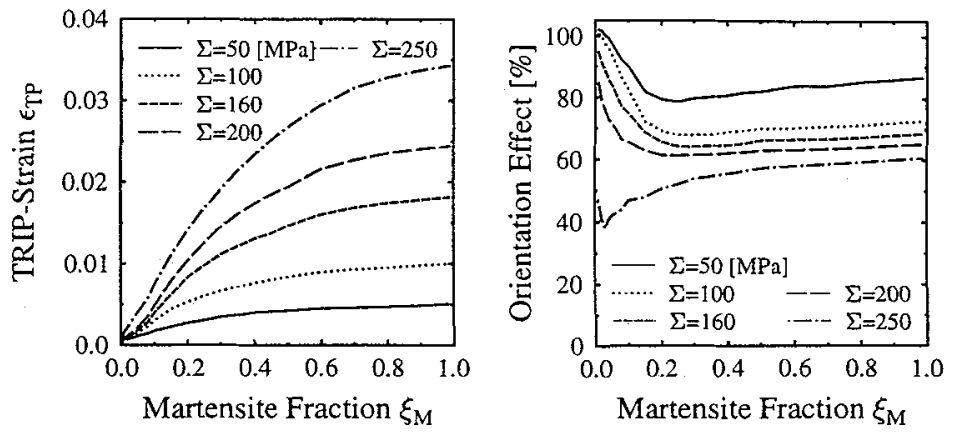

Figure 1: Left a): TRIP-strain $\epsilon_{T P}$ in dependence of volume fraction $\xi_{M}$ for different levels of $\Sigma$. Right b): Percentage of orientation effect contributing to overall TRIP-strain.

Furthermore, the proposed modelling approach allows a quantitative study of the microstructure development by computing the volume fractions of transformed variants $\xi_{M, j}^{k}$. With respect to material design efforts the development of a controlled microstructure by thermomechanical loading is desired. In the case here this would mean the transformation of a certain limited number of martensitic variants to give an orientation texture. Fist we select three representative crystallites of the mesodomain with respect to their orientation to the direction of applied stress. Therefore we calculate the Schmid-factors $m_{j}^{k}$ for the transformation shear $\gamma$ of the $24 \times \mathrm{N}$ possible variants. Within each crystallite the maximum Schmid-factor $m_{\text {max }}^{k}$ is determined. Then, from those $\mathrm{N}$ values we determine the crystallites with the maximum $m_{\text {max }}^{*}=0.495$, minimum $m_{m i n}^{*}=0.190$ and average $m_{a v}^{*}=0.412$ Schmid-factor. For the crystallite with an average orientation the numbers of transformed variants are depicted in Figure 2a in dependence of the applied stress level and the transformation progress. In the early stage of the MT only a limited number of variants transforms. As MT starts variants with high Schmid-factors transform but not that ones with $m_{\max }^{k}$ due to the effect of internal stresses. Since with increasing $\xi_{M}$ the chemical driving force dominates over the mechanical driving forces a larger number of variants are able to transform simultaneously. With increasing $\Sigma$ the effect of variant selection is increasing but decreases if the mesodomain is loaded near the yield stress of the parent phase $\left(\sigma_{Y, f c c}=250[M P a]\right)$. The microstructure development may depend on the orientation of the grains as shown in Figure $2 \mathrm{~b}$. In a favorably oriented crystallite the selection effect is completed at $\xi_{M}=0.2$ in contrast to the average case. In a least favorable oriented crystallite the the situation is similar. Transformation of specific variants does not occur in that case. The results show that the OE cannot be neglected in macroscopic constitutive laws for TRIP as already stated in Ref. [9]. Secondly, a controlled microstructural rearrangement does not seem to be possible for a material where the AE as a significant microscale deformation mechanism is apparent.
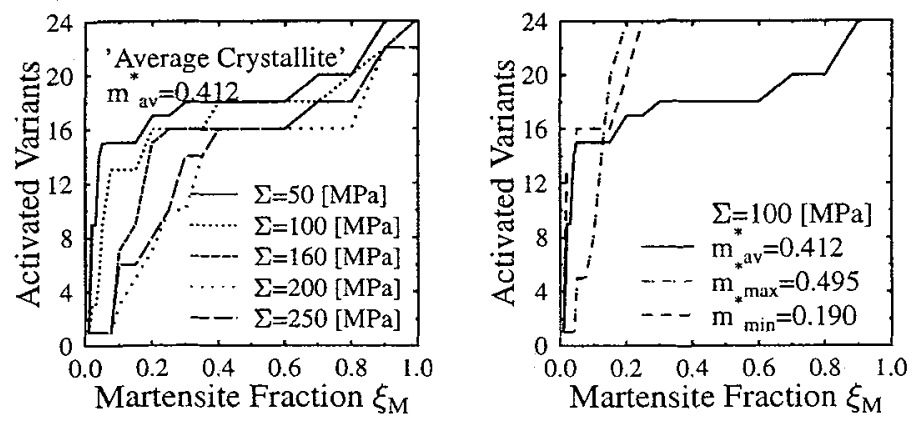

Figure 2: Left a): Number of transformed variants in a crystallite with average maximum Schmid factor $m_{a v}^{*}$ in dependence of $\xi_{M}$ and applied stress level $\Sigma$. Right b): Number of transformed variants for 3 crystallites 
with average $\left(m_{a v}^{*}\right)$, most favorable $\left(m_{m a x}^{*}\right)$ and least favorable orientation $\left(m_{m i n}^{*}\right)$.

\section{MICROMECHANICS of VARIANT COALESCENCE}

\subsection{One-Way Shape Memory Effect}

The mechanical behavior of shape memory alloys (SMA) under uniaxial tensile load stress ( $\Sigma=\Sigma \boldsymbol{\lambda}, \lambda_{l m}=$ 0 except $\lambda_{33}=1$ ) shows a large variety depending on material's microstructure. The mechanisms of microstructural changes result directly in important features of the stress-strain curves dependent on temperature. The process of variant coalescence is one of successive steps classifying the one-way shape memory effect to be shortly recalled : First a parent $\rightarrow$ martensite transformation upon cooling under zero externally-applied stress $(\dot{T}<0, \Sigma=0)$ occurs where the shape of a specimen remains unchanged due to a self-accommodation process (no $\mathrm{OE}$ ). The second step is the process of variant coalescence in the fully martensitic state upon stressing $(\dot{T}=0, \Sigma \neq 0$ ) followed by unloading and, finally, by the reverse martensite $\rightarrow$ parent transformation $(\dot{T}>0, \Sigma=0)$ to restore the specimen to its original shape.

\subsection{Kinematical Description of Self-Accommodation and Reorientation}

In a temperature regime $T>M_{s}$ the mesodomain contains the $\beta_{1}$-parent phase only $\left(\xi_{M}=0\right)$. Due to $\dot{T}<0$ the $\beta_{1} \rightarrow \gamma_{1}^{\prime}$ transformation takes place. The WLR-theory predicts a $\{41112\}_{\beta_{1}}$ habit plane type in $\mathrm{Cu}-\mathrm{Al}-\mathrm{Ni}$ alloys proved experimentally by Otsuka et al. [13]. The material input data for the kinematical description are listed in Ref.[14]. Below $M_{f}$ the mesodomain is in the fully martensitic state. The $\beta_{1} \rightarrow \gamma_{1}^{\prime}$ transformation occurs in a self-accommodating manner. A morphology with diamond-shaped plate groups $M_{k}, M_{k}=1,6$ consisting of four variants clustering around the six $\left\{\begin{array}{lll}1 & 1 & 0\end{array}\right\}_{\beta_{1}}$ poles appear, hence there are six plate groups $M_{k}$ within each crystallite $k$. A process coordinate for the process of variant coalescence under consideration $z, 0 \leq z \leq 1$, is introduced. Let us define the initial self-accommodating state at $z=0$. Now a load stress $\Sigma$ is applied to the mesodomain. The assumption is made that each of the plate groups $M_{k}$ appears with the same volume fraction $\xi^{M_{k}}=f_{k} / 6$. Furthermore, we state that in the fully self-accommodating state each four variants $A^{M_{k}}, B^{M_{k}}, C^{M_{k}}$ and $D^{M_{k}}$ of any plate group appears with the same volume fraction as depicted in Figure $3 \mathrm{a}$,

$$
\xi_{A}^{M_{k}}(0)=\xi_{B}^{M_{k}}(0)=\xi_{C}^{M_{k}}(0)=\xi_{D}^{M_{k}}(0)=\frac{\xi^{M_{k}}}{4}=\frac{f_{k}}{24} .
$$
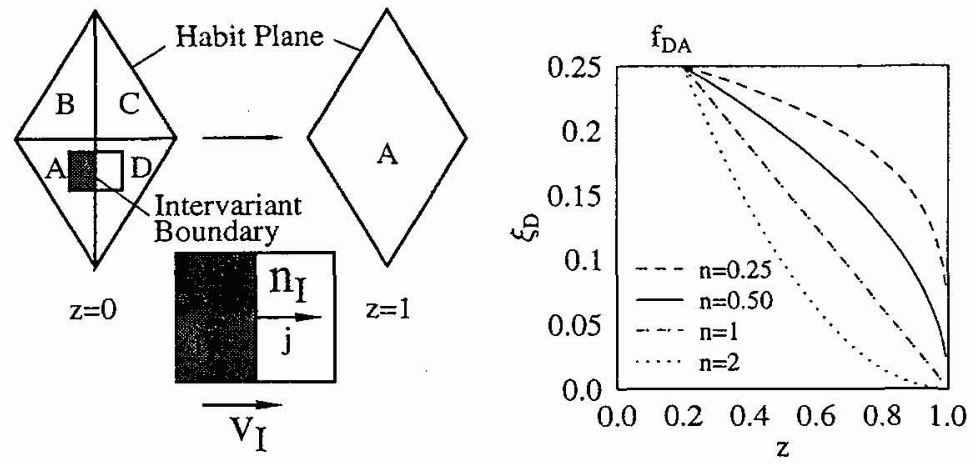

Figure 3: Left a): Definition of initial self-accommodating state and final state after complete reorientation within a plate group. Right $\mathrm{b}$ ): Microkinetical formulation of the reorientation process $D \rightarrow A$ : Normalized volume fraction of reorienting variant $D$.

A single variant eventually results as a consequence of the reorientation process. Let us also define the microstructure corresponding to the final state of reorientation in the mesodomain at $z=1$. In each plate 
group $M_{k}$ only the most favorable variant $A^{M_{k}}$ in a thermodynamical sense prevails as depicted in Figure $3 \mathrm{a}$ to yield the largest transformation strain in the direction of $\Sigma$ so that

$$
\xi_{A}^{M_{k}}(1)=\frac{f_{k}}{6}, \quad \xi_{B}^{M_{k}}(1)=\xi_{C}^{M_{k}}(1)=\xi_{D}^{M_{k}}(1)=0 .
$$

\subsection{Process Description of Variant Coalescence}

\subsubsection{Condition for Thermodynamic Admissibility}

The $\mathrm{AE}$ as a result of microscale plastic deformations does not play any crucial role in shape memory alloys. In the case considered here the internal strain energy $U_{e, \text { int }}$ due to reorientation-induced internal stresses has an important influence on the macroscopic mechanical behavior. The energy changes in the case of shape memory alloys can be classified in terms known as the chemical free energy, elastic energy and interfacial energy due to grain boundaries $\Gamma_{I B}$ and boundaries between martensitic variants $\Gamma_{G B}$. Taking homogeneous elastic material behavior for the $\gamma_{1}^{\prime}$-phase the Gibbs free energy for the quantitative description of the state of the polycrystalline mesodomain is written in the form

$$
G=\phi_{C}(\theta)+\Gamma_{G B}+\Gamma_{I B}+U_{e, i n t}-U_{e, 0}-\left(\Sigma: \mathbf{E}_{T}\right) V
$$

for a derivation see Ref.[14]. $U_{e, 0}$ is the strain energy due to $\Sigma$. The increment in Gibbs free energy for the reorientation process occurring isothermally by discarding the increment of intervariant boundary energy $\dot{\Gamma}_{I B}$ results as

$$
\dot{G}=\dot{U}_{e, i n t}-\dot{U}_{e, 0}-\left(\dot{\Sigma}: \mathbf{E}_{T}\right) V-\left(\Sigma: \dot{\mathbf{E}}_{T}\right) V .
$$

The general principles to be used for the derivation of the condition for thermodynamic admissibility (CTA) are the principle of virtual work and the local forms of first law and second law of thermodynamics. For a detailed derivation see Ref. [14]. Variant coalescence is considered as a slow process so that inertia terms in the global balances of momentum can be disregarded and the contribution of kinetic energy is omitted in the global statement of the first law of thermodynamics. Across the boundaries between the martensitic variants forming plate groups thermomechanical quantities suffer jump discontinuities. We shall introduce the concept of a scalar driving traction $F_{I}$ on an interface having been developed by several authors, see Refs. [15], [16], [17]. Due to a homogeneous temperature distribution in the mesodomain and elastic material behavior the contributions from heat conduction and local dissipation in the material away from the interfaces to the total rate of entropy production vanish. The only contribution comes from moving intervariant boundaries. Starting from the local form of Gibbs free energy, invoking the principle of virtual work and the Hill-Mandel principle having body forces neglected, taking intervariant boundaries to be coherent and neglecting inertia terms the increment in Gibbs free energy $G$ takes the form

$$
\dot{G}=-V \dot{\boldsymbol{\Sigma}}: \mathbf{E}-\dot{\Phi}_{I}
$$

for a derivation see Ref. [14]. $\dot{\Phi}_{I} \geq 0$ is the total rate of dissipation for the mesodomain due to entropy production from all the moving intervariant boundaries. The inequality is the consequence from the local forms of the second law of thermodynamics.

By taking eq. (11) and eq. (12) one finally arrives at the CTA for variant coalescence to be written as

$$
\left(\Sigma: \dot{\mathbf{E}}_{T}\right) V=\dot{U}_{e, \text { int }}+\dot{\Phi}_{I}
$$

For the energy dissipation associated with the reorientation of variants we follow Sun [18] to take the simple constitutive assumption that the rate of dissipation for the mesodomain $\dot{\Phi}_{I}$ is proportional to the overall rate of volume fraction of reorientation $\dot{\Phi}_{I}=D^{r e} \dot{\xi}^{r e}$, where $D^{r e}>0$ is assumed to be a constant. Three variants $B^{M_{k}}, C^{M_{k}}$ and $D^{M_{k}}$ in each of the self-accommodating plate groups $M_{k}$ convert into the most favorable remaining variant within the group $A^{M_{k}}$. $\xi^{r e}$, therefore, directly results from the reorientation of variants within each of the plate groups and is obtained by summing over the rates of microfractions $\dot{\xi}_{D \rightarrow A}^{M_{k}}$, $\dot{\xi}_{C \rightarrow A}^{M_{k}}$ and $\dot{\xi}_{B \rightarrow A}^{M_{k}}$. By inserting for $\dot{\Phi}_{I}$ the CTA is now written as

$$
\left(\Sigma: \dot{\mathbf{E}}_{T}\right) V=\dot{U}_{e, \text { int }}+D^{r e} \dot{\xi}^{r e} .
$$




\subsubsection{Constitutive Assumptions for the Behavior of Self-Accommodating Microstructures}

The objective of the modelling procedure in this article is to predict the overall mechanical behavior by the stress-strain curve (model output) as a result of applying simple constitutive formulations describing the reorientation process in the plate groups by a quantitative micromechanics approach. As model input we start from a kinematical description of the self-accommodating microstructure. The kinematical description of the reorientation process is performed by transformation strain tensors corresponding to martensitic variants. For the microstructural rearrangements we make simple reasonable assumptions. The start of the reorientation process between two variants $j$ and $j^{*}$ is taken to be governed by the normalized driving traction (NDT) $F_{I, j \rightarrow j^{*}}^{M_{k}}=\boldsymbol{\lambda}:\left(\epsilon_{T, j^{*}}^{k}-\epsilon_{T, j}^{k}\right)$, which is computed for each of the $6 \times N$ plate groups. A 'heapsort'-routine is applied to sort the 3 NDT-values. The most favorable variant giving the largest transformation strain in the direction of $\Sigma$ is labelled $A^{M_{k}}$. The three converting variants are given labels $B^{M_{k}}, C^{M_{k}}$ and $D^{M_{k}}$. The corresponding NDTs for the reorientation of $D^{M_{k}} \rightarrow A^{M_{k}}, C^{M_{k}} \rightarrow A^{M_{k}}$ and $B^{M_{k}} \rightarrow A^{M_{k}}$ are written

$$
F_{D \rightarrow A}^{M_{k}}=\lambda:\left(\epsilon_{T, A}^{M_{k}}-\epsilon_{T, D}^{M_{k}}\right), F_{C \rightarrow A}^{M_{k}}=\lambda:\left(\epsilon_{T, A}^{M_{k}}-\epsilon_{T, C}^{M_{k}}\right), F_{B \rightarrow A}^{M_{k}}=\lambda:\left(\epsilon_{T, A}^{M_{k}}-\epsilon_{T, B}^{M_{k}}\right),
$$

thus $F_{D \rightarrow A}^{M_{k}} \geq F_{C \rightarrow A}^{M_{k}} \geq F_{B \rightarrow A}^{M_{k}}>0$. That means, reorientation starts first for the least favorable variant $D^{M_{k}}$, then for $C^{M_{k}}$ and finally for $B^{M_{k}}$. Additionally, we look for the plate group $M_{k}^{*}$ where the reorientation process starts first at $z=0$, that means for the maximum NDT in the polycrystal $F^{*}=F_{D \rightarrow A}^{M_{k}^{*}}$. The lower the NDT is, the higher is $\Sigma$ required for the activation of the reorientation process. Let us introduce for each $M_{k}$

$$
f_{D A}^{M_{k}}=1-\frac{F_{D \rightarrow A}^{M_{k}}}{F^{*}}, f_{C A}^{M_{k}}=1-\frac{F_{C \rightarrow A}^{M_{k}}}{F^{*}}, f_{B A}^{M_{k}}=1-\frac{F_{B \rightarrow A}^{M_{k}}}{F^{*}}
$$

so that $0 \leq f_{D A}^{M_{k}} \leq f_{C A}^{M_{k}} \leq f_{B A}^{M_{k}} \leq 1$. The higher the NDT the 'earlier' the reorientation process will take place within the process interval $0 \leq z \leq 1$. The reorientation process in a plate group is activated if

$$
z=f_{D A}^{M_{k}}, z=f_{C A}^{M_{k}}, \quad \text { and } \quad z=f_{B A}^{M_{k}}
$$

In the case that $z<f_{D A}^{M_{k}}, z<f_{C A}^{M_{k}}$ or $z<f_{B A}^{M_{k}}$ there is no reorientation process between the corresponding variants. Once the reorientation process between two variants in a plate group $M_{k}$ is activated the process takes place the way that the converting variants $D^{M_{k}}, C^{M_{k}}$ and $B^{M_{k}}$ transform into variant $A^{M_{k}}$ gradually dependent on the intervariant boundary mobility as depicted in Figure $3 \mathrm{~b}$. The microkinetics relation for the conversion $D^{M_{k}} \rightarrow A^{M_{k}}$ in the region covered by $D^{M_{k}}$ is written as

$$
\xi_{D}^{M_{k}}(z)=\left\{\begin{array}{cll}
\frac{f_{k}}{24} & \text { if } & z<f_{D A}^{M_{k}} \\
\frac{f_{k}}{24}\left(\frac{1-z}{1-f_{D A}^{M_{k}}}\right)^{n} & \text { if } & z \geq f_{D A}^{M_{k}}
\end{array}\right.
$$

For $C^{M_{k}} \rightarrow A^{M_{k}}$ and $B^{M_{k}} \rightarrow A^{M_{k}}$ only labels change. The reorientation process for case $A$ is finished at the same instant at $z=1$ within each plate group. The influence of the intervariant boundary mobility is taken to be considered via the exponent $n$. An increasing $n$ corresponds to an increasing interface mobility. In the limit case $n \rightarrow \infty$ a variant would be reoriented as a whole by a jump once activated which corresponds to a nucleation-type process. The reorientation history in dependence of $n$ is schematically depicted in Figure $3 \mathrm{~b}$ taking the normalized volume fraction of a plate group to be $\tilde{\xi}^{M_{k}}=1$ so that the volume fraction of rearranging variant $D^{M_{k}}$ at $z=0$ is $\tilde{\xi}_{D}^{M_{k}}(0)=0,25$. At the end of the process only the variant $A^{M_{k}}$ prevails so that $\tilde{\xi}_{D}^{M_{k}}(0)=0$.

\subsection{Micromechanical Modelling Framework}

The kinematical description of the reorientation process in incremental form is now specified for each crystallite $k$ by averaging over the six plate groups with variants $A^{M_{k}}, B^{M_{k}}, C^{M_{k}}$ and $D^{M_{k}}$ by

$$
\dot{\mathbf{E}}_{T}^{k}(z)=\frac{1}{f_{k}} \sum_{M_{L}=1}^{6} \sum\left[\dot{\xi}_{A}^{M_{k}}(z) \epsilon_{T, A}^{M_{k}}+\dot{\xi}_{B}^{M_{k}}(z) \epsilon_{T, B}^{M_{k}}+\dot{\xi}_{C}^{M_{k}}(z) \epsilon_{T, C}^{M_{k}}+\dot{\xi}_{D}^{M_{k}}(z) \epsilon_{T, D}^{M_{k}}\right]
$$


The finite element analysis is carried out to calculate the increments of strain energy $U_{e, \text { int }}$ stored in the mesodomain due to the elastic accommodation of $\dot{\mathbf{E}}_{T}^{k}(z)$. The process is simulated by imposing for each crystallite $\dot{\mathbf{E}}_{T}^{k}(z)$ which causes an internal reorientation-induced internal stress state $\boldsymbol{\sigma}_{\text {int }}(\underline{r}, z)$ to develop with the corresponding strain energy density $U_{e, i n t}^{V}(\underline{r}, z), \underline{r}$ is the position vector. At each instant $z$ the stored internal strain energy $U_{e, \text { int }}(z)$ and the corresponding increment $\dot{U}_{e, \text { int }}(z)$ is calculated for the entire mesodomain. By averaging over the mesodomain we can also determine $\mathbf{E}_{T}(z)$ with the corresponding increment $\dot{\mathbf{E}}_{T}(z)$ and the increment of reorienting volume fraction $\dot{\xi}^{r e}(z)$. Finally, we have to quantify the energy dissipation $\dot{\Phi}_{I}$ by the constant $D^{r e}$. Then for a certain increment of the reorientation process the required externally-applied stress $\Sigma$ can be calculated from the thermodynamic condition (14). If the thermodynamic condition is fulfilled the increment of the overall mechanical driving force $\Sigma: \dot{\mathbf{E}}_{T} V$ compensates the internal strain energy and the energy dissipation. The macroscopic strain $\mathbf{E}$ is calculated as a sum of transformation strains and elastic strains $\mathbf{E}=\mathbf{E}_{T}+\mathbf{E}_{e}$. This way the macroscopic mechanical behavior can be determined by incremental construction of the stress-strain curve.

\subsection{Results and Discussion}

The influence of the intervariant boundary mobility described via the exponent $n$ in the microkinetics formulation is considered in this article. For increasing boundary mobility (increasing $n$ ) we observe drops in the stress-strain curves as shown in Figure 4a. In that situation the reorientation process occurs without any increase of $\Sigma$ but due to a release in internal elastic energy $U_{e, \text { int }}$. If during an increment $\Delta z$ the shape change due to the reorientation process is elastically accommodated so that $\dot{U}_{e, i n t}>0$ we call it a process of elastic accommodation. The drops in the stress-strain curve occur if $\dot{U}_{e, \text { int }}<0$, what we then should call an unaccommodation processes. The higher the boundary mobility is the higher will be the probability for unaccommodation processes and serrations in the stress-strain curve.
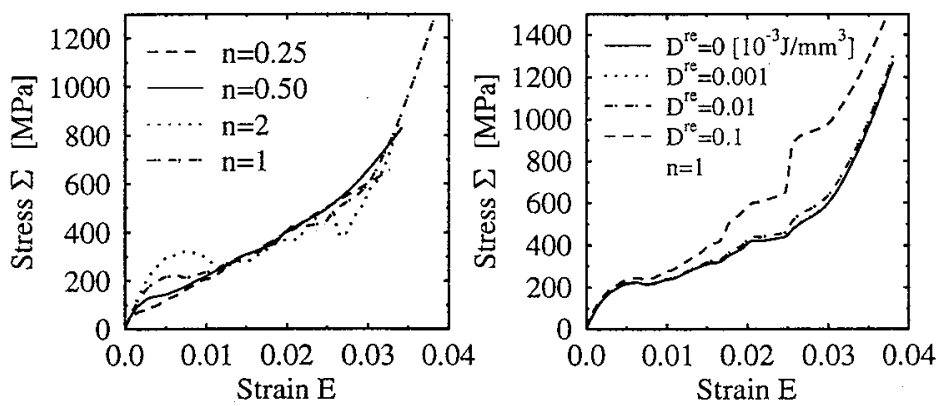

Figure 4: Left a): Macroscopic mechanical behavior under variant coalescence. Effect of intervariantboundary mobility. Right $b$ ): Effect of energy dissipation during the reorientation process for $n=1$.

Next, the effect of energy dissipation due to interfacial motion is taken into account by variation of the value for the constant $D^{r e}$. The stress-strain curves depicted for $n=1$ in Figure $4 \mathrm{~b}$ are shifted to higher stress levels with increasing energy dissipation as can be also immediately concluded from eq.(14). With increasing $D^{r e}$ one can also expect changes in the shape of the stress-strain curves. If $n \rightarrow \infty$ the reorientation process within a plate group occurs like a nucleation-type process. In that case accommodation and unaccommodation processes can easily alternate leading to strong serrations in the stress-strain curves. Serrations in the stress-strain curves have been experimentally observed for single crystal specimens under $\mathrm{VC}$ [13]. It has been concluded that the rapid movement of interfaces causes the sharp stress drops in the stress-strain curves. This phenomenon is captured by the modelling procedure adopted here well although it is not reported to occur in experiments on polycrystalline specimens. 


\section{References}

[1] E. Kröner: GAMM-Mitteilungen, 1992, vol. 2, pp. 104-119.

[2] E. Gautier, X.M. Zhang and A. Simon, Proc. $2^{\text {nd }}$ Int. Conf. Residual Stresses, G. Beck, S. Denis and A. Simon Eds. (Elsevier, London, 1989) pp. 777-783.

[3] J.R. Patel and M. Cohen, Acta Metall. 1 (1953) pp. 531-538.

[4] C.L. Magee, The Nucleation of Martensite, in: Phase Transformations, H.I. Aaronson Ed. (ASM, Warrendale, PA, 1968) pp. 115-156.

[5] M.S. Wechsler, D.S. Lieberman and T.A. Read, J. Metals, Trans. AIME 197 (1953) pp. 1503-1515.

[6] F.D. Fischer, M. Berveiller, K. Tanaka and E.R. Oberaigner, Arch. Appl. Mech., 64 (1994) pp. 54-85.

[7] Marketz F. and Fischer F.D., Modelling Simul. Mater. Sci. Eng. 5 (1994) pp. 1017-1046.

[8] Marketz F. and Fischer F.D., Proc. Int. Conf. Solid-Solid Phase Transformations, Johnson, W.C., Hoẉe, J.M., Laughlin, D.E. and Soffa, W.A. Eds. (Metall. Soc. AMME, Warrendale, PA, 1994) pp. 785-790.

[9] Marketz, F. and Fischer, F.D., Metall. Trans. A 26A (1995) pp. 267-278.

[10] J.R. Rice, in: Constitutive Equations in Plasticity, A.S. Argon Ed. (The MIT Press, Cambridge, Massachusetts, 1975) pp. 23-78.

[11] G.B. Olson, in: Deformation, Processing and Structure, G. Krauss Ed. (ASM, Warrendale, PA, 1984) pp. 391-424.

[12] G.B. Olson, K. Tsuzaki and M. Cohen, in: Phase Transitions in Condensed Systems, G. Carhill, F. Spaepen and K.N. Tu Eds., Mat. Res. Soc. Symp. Proc. (1987) pp. 129-148.

[13] K. Otsuka, C.M. Wayman, K. Nakai, H. Sakamoto and K. Shimizu: Acta Metall. 24 (1975) pp. 207-226.

[14] F. Marketz and F.D. Fischer, Comput. Mat. Sci. (1995) to be published.

[15] R. Abeyaratne and J.K. Knowles, J. Mech. Phys. Solids 38 (1990) pp. 345-360.

[16] W. Heidug and F.K. Lehner, PAGEOPH 123 (1985) pp. 91-98.

[17] M. Berveiller, E. Patoor and M. Buisson, J. Phys. IV, Coll. C4, suppl. J. Phys III 1 (1991) pp. 387-396.

[18] Q.P. Sun and K.C. Hwang, J. Mech. Phys. Solids 41 (1993) pp. 1-17.

\section{Acknowledgements}

We gratefully acknowledge valuable discussions with Professor M. Berveiller, LPPM, I.S.G.M.P., Metz. The ABAQUS finite element code was made available under academic licence from Hibbitt, Karlsson and Sorensen Inc., Pawtucket, RI, USA. 